\title{
Coating for High-Magnification with Your Au/Pd Sputter Coater.
}

\author{
K. Pham
}

Medtronic Inc., 7000 Central Ave. NE, Minneapolis, MN 55432

In an era of reduced spending, we are often being asked to be more creative with the equipment that we are already have. In this study we wanted to show that you can achieve similar resolution with ion beam platinum $(\mathrm{Pt})$ sputter coating and gold $(\mathrm{Au})$-palladium $(\mathrm{Pd})$ magnetron triode sputter coating. Au-Pd coating is usually resolved above 20,000x while Pt ion beam coating can be resolved above $100,000 x$. A typical ion beam sputter coater costs approximately $\$ 50,000$ and our plasma-magnetron sputter coater cost around $\$ 7,000$.

Commercial stretched polypropylene microporous hollow fiber is commonly found in surgical medical equipment. To examine the fiber using a scanning electron microscope (SEM) it needs to be coated to provide an electrically conductive layer. This suppresses surface charging and minimizes radiation damage from the electron beam. The coating is intended to be a thin replica of the specimen surface.

Sample preparation: The fibers were mounted on an aluminum stub with a conductive carbon adhesive pad then pained at each end of the fiber with silver paint to improve conductivity. One control sample was not coated. The second sample was coated in a magnetron triode sputter coater with Au-Pd for 5 minutes at $1 \mathrm{~mA}$ and 5volts. We try to apply as little coating as possible. Another set of fibers was coated with a single ion beam sputter coater with Pt for 5 minutes at $2 \mathrm{~mA}$ and $5 \mathrm{KV}$.

The imaging of the stretched polypropylene microporous hollow fibers was done in a Hitachi S4500 FESEM operating at $1 \mathrm{kV}$ and $5 \mathrm{~mm}$ working distance. The porous structures on the uncoated sample were distorted due to a build up of electrical charge on the surface (Figure 1). Au-Pd grain structure was not visible on the fiber surface (Figure 2). The porous structure appeared similar to the structure in the Pt coated fiber (Figure 3).

Optimizing sputter machine coating thickness and distribution ${ }^{[1]}$ can extend the effective magnification of Au-Pd coating.

${ }^{[1]}$ Chapman, S. Microscopy Today, November 2001, 28. 


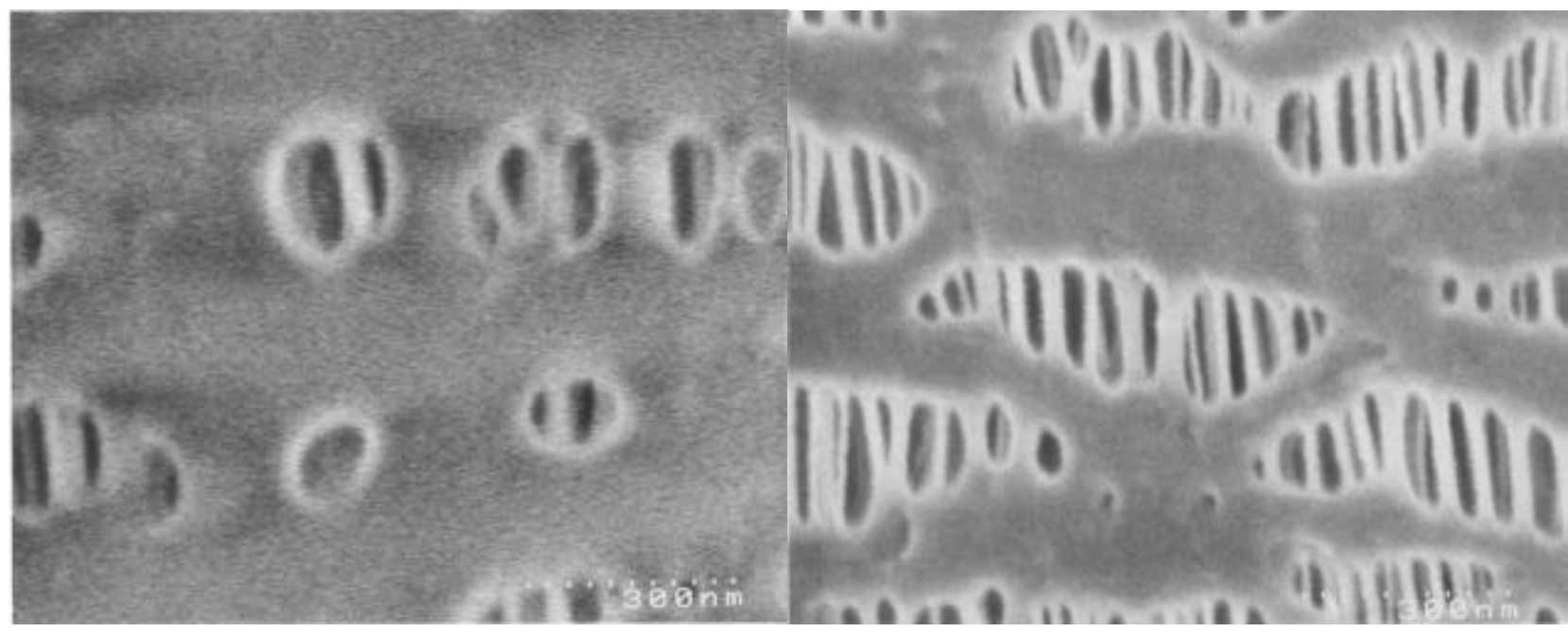

FIG. 1. Uncoated stretched polypropylene microporous hollow fiber.
FIG. 2. Au-Pd coated stretched polypropylene microporous hollow fiber.

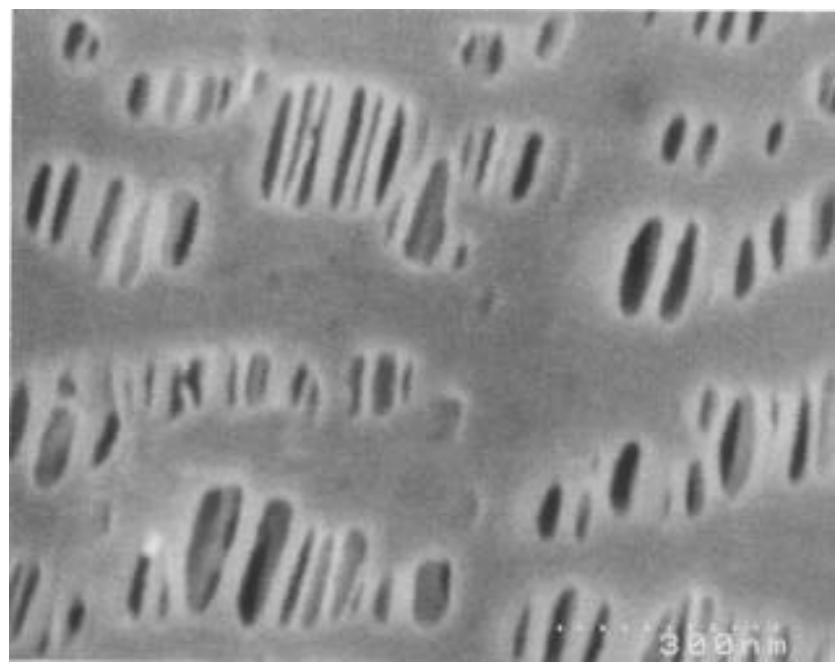

FIG. 3. Pt coated stretched polypropylene microporous hollow fiber.. 